\title{
AN APPLICATION OF COMPLEX BORDISM TO THE STABLE HOMOTOPY GROUPS OF SPHERES
}

\author{
BY LARRY SMITH
}

Communicated by Edgar H. Brown, October 30, 1969

In this note we announce several results that are a continuation of the study initiated in [1] of the internal properties of the complex bordism homology functor applied to finite complexes and their external applications. The present work is an outgrowth of our attempts to better understand the results of $[1]$ and [2], especially $[1, \S 6]$ and $[2, \S \S 2-3]$ that deal with the study of

$$
\operatorname{Im}\left\{\Omega_{*}^{f r}(X) \rightarrow \Omega_{*}^{U}(X)\right\}
$$

$X$ a finite complex. This has brought us into contact with many new and interesting questions concerning the realizability of certain cyclic $\Omega_{*}^{U}$-modules as complex bordism modules of finite complexes. Needless to say it has also involved us in the stable homotopy groups of spheres, particularly with the occurrence of certain types of infinite families of elements in the $p$-component (see the discussion below).

The particular $\Omega_{*}^{U}$-modules that are of interest to us will require some preparation to describe. Let us therefore fix an odd prime integer $p$. We recall that there are $U$-manifolds $V^{2 p^{i}-2}$, of dimension $2 p^{i}-2$, all of whose mod $p$ Chern numbers vanish, but are acceptable polynomial generators for $\Omega_{*}^{U}$ in dimension $2 p^{i}-2$ [3], [4]. What we seek is for each nonnegative integer $n$ a finite complex $V(n)$ such that

$$
\tilde{\Omega}_{*}^{U}(V(n)) \cong \Omega_{*}^{U} /\left(p,\left[V^{2 p-2}\right], \cdots,\left[V^{2 p^{n}-2}\right]\right)
$$

as an $\Omega_{*}^{U}$-module.

For $n=0$ we may choose for the space $V(0)$ the Moore space $S^{1} \cup_{p} e^{2}$ where $p: S^{1} \rightarrow S^{1}$ is a map of degree $p$. We then know (see e.g. $[2, \S 3])$ that

$$
\operatorname{Im}\left\{\tilde{\Omega}_{*}^{f r}(V(0)) \rightarrow \tilde{\Omega}_{*}^{U}(V(0))\right\}
$$

consists of the subgroup generated by the elements

$$
\left.\left\{\left[V^{2 p-2}\right]\right]_{\sigma} \mid t=0,1, \cdots\right\}
$$

AMS Subject Classifications. Primary 5545, 5710.

Key Words and Phrases. Stable homotopy groups, complex bordism modules, spherical bordism classes, Toda brackets. 
where $\sigma \in \tilde{\Omega}_{1}^{U}\left(S^{1} \cup_{p} e^{2}\right)$ is the canonical generator. Thus for each integer $t \geqq 0$ we obtain stable mappings

$$
\phi_{t}: S^{2 p t-2 t+1} \rightarrow S^{1} \cup_{p} e^{2}
$$

where

$$
\left[S^{2 p t-2 t+1}, \phi_{t}\right]=\left[V^{2 p-2}\right] t^{t}
$$

in $\tilde{\Omega}_{*}^{U}\left(S^{1} \cup_{p} e^{2}\right)$.

THEOREM I. With the above notations the composite

$$
q \phi_{t}: S^{2 t(p-1)+1} \underset{\phi_{t}}{\rightarrow} S^{1} \cup_{p} e^{2} \underset{q}{\rightarrow} S^{2}
$$

is stably essential for each $t=1,2, \cdots$; where $q$ denotes the natural quotient mapping. We thus obtain elements

$$
\left[q \phi_{t}\right] \neq 0 \in \stackrel{s}{\pi_{2 t(p-1)-1}}, \quad t=1,2, \cdots
$$

which may be seen to be of order $p$. (Here we have written $\pi_{*}^{S}$ for $\Omega_{*}^{f r}$ the stable homotopy ring of spheres.)

A detailed comparison of the proof of this result with the constructions of [5] brings forth the equality

$$
\left[q \phi_{t}\right]=\alpha_{t}, \quad t=1,2, \cdots
$$

where $\left\{\alpha_{t}\right\}$ is the infinite family of elements introduced by Toda [5]. As the mapping

$$
\phi=\phi_{1}: S^{2 p-1} \rightarrow V(0)
$$

has $\operatorname{order} p$ it may be extended to a mapping

$$
\tilde{\phi}: S^{2 p-1} \wedge V(0) \rightarrow V(0),
$$

and one easily obtains:

TheOREM II. With the above notation let $C(\tilde{\phi})$ denote the mapping cone of $\tilde{\phi}$. Then

$$
\Omega_{*}^{U}(C(\tilde{\phi})) \cong \Omega_{*}^{U} /\left(p,\left[V^{2 p-2}\right]\right) .
$$

Thus for the space $V(1)$ we may select $C(\tilde{\phi})$. Our next task is to take up the study of

$$
\operatorname{Im}\left\{\tilde{\Omega}_{*}^{f r}(V(1)) \rightarrow \Omega_{*}^{U}(V(1))\right\} .
$$

With the aid of the Adams spectral sequence we obtain: 
THEOREM III. Let $p$ be a prime, $p>3$. Then with the above notation, there exists for each nonnegative integer $t$ a stable map

$$
\psi_{t}: S^{2 t\left(p^{2}-1\right)+1} \rightarrow V(1)
$$

such that

$$
\left.\left[S^{2 t\left(p^{2}-1\right)+1}, \psi_{t}\right]=\left[V^{2 p^{2}-2}\right]\right]^{t} \sigma
$$

in $\tilde{\Omega}_{*}^{U}(V(1))$, where $\sigma \in \tilde{\Omega}_{1}^{U}(V(1))$ is the canonical class.

The space $V(1)$ is seen to have a stable cell structure

$$
V(1)=S^{1} \cup_{p} e^{2} \cup_{\phi} e^{2 p} \cup_{p} e^{2 p+1} .
$$

Let

$$
q: V(1) \rightarrow S^{2 p+1}
$$

denote the natural quotient mapping.

The following theorem then constitutes our main result.

TheOREM IV. Let $p$ be a prime, $p>3$. Then with the above notations the composite

$$
q \psi_{t}: S^{2 t\left(p^{2}-1\right)+1} \underset{\psi_{t}}{\rightarrow} V(1) \underset{q}{\rightarrow} S^{2 p+1}
$$

is stably essential for $t=1,2, \cdots$. We thus obtain elements

$$
\left[q \psi_{t}\right] \neq 0 \in \stackrel{s}{\pi_{2 p(t p-1)-2 t},} \quad t=1,2, \cdots
$$

which are of order $p$.

Upon observing that

$$
2 p(t p-1)-2 t=2(p-1)(t p+t-1)-2
$$

it is natural to conjecture that (appropriately constructed) the elements $\left\{\left[q \psi_{t}\right]: t=1, \cdots\right\}$ generalize the elements $\left\{\beta_{t}: t=1, \cdots, p+1\right\}$ introduced by Toda in [5] when $p>3$. (The elements $\left[q \psi_{1}\right]$ and $\beta_{1}$ may be seen to coincide because $\pi_{2_{p}(p-1)-2}^{S}(p) \cong Z_{p}$ with generator $\left.\beta_{1}[5].\right)$

The mapping, for $p>3$,

$$
\psi=\psi_{1}: S^{2 p^{2}-1} \rightarrow V(1)
$$

is shown to extend to a mapping

$$
\tilde{\psi}: S^{2 p^{2}-1} V(1) \rightarrow V(1)
$$

and we obtain: 
THEOREM V. With the above notation let $C(\tilde{\psi})$ denote the mapping cone of $\tilde{\psi}$. Then for all primes $p, p>3$, we have

$$
\tilde{\Omega}_{*}^{U}(C(\tilde{\psi}))=\Omega_{*}^{U} /\left(p,\left[V^{2 p-2}\right],\left[V^{2 p^{2}-2}\right]\right) .
$$

Thus for the space $V(2)$ we may select $C(\tilde{\psi})$ whenever $p$ is a prime strictly greater than 3 . In view of our discussion above we should next take up the study of

$$
\operatorname{Im}\left\{\tilde{\Omega}_{*}^{f r}(V(2)) \rightarrow \tilde{\Omega}_{*}^{U}(V(2)) .\right.
$$

It is quite tempting to conjecture that this image is generated by the classes

$$
\left\{\left[V^{2 p^{3}-p}\right]^{t} \sigma \mid t=0,1, \cdots\right\}
$$

as an abelian group when $p$ is a suitably chosen prime, say $p>5$. We have however been unable to establish this and are therefore unable at present to construct a space $V(3)$ for any prime. The situation for $p=2$ is exceptional and is discussed in [2]. The situation for $p=3$ becomes exceptional in the study of

$$
\operatorname{Im}\left\{\Omega_{*}^{f r}(V(1)) \rightarrow \Omega_{*}^{U}(V(1))\right\} .
$$

We may construct $\psi_{1}$ for $p=3$ but it does not extend to $\tilde{\psi}$. Nor do we know which $\psi_{t}$ may be constructed.

The existance of further spaces $V(3), V(4), \cdots$ in the series would seem to imply the presence of further infinite families of elements in the $p$-component of the stable homotopy groups of spheres. This is a point that deserves further scrutiny and is at present under active investigation.

\section{REFERENCES}

1. P. E. Conner and L. Smith, On the complex bordism of finite complexes, Inst. Hautes Etudes Sci. Publ. Math. No. 37 (1970).

2. P. E. Conner and L. Smith, Homological dimensions of complex bordism modules, Proceedings of the 1969 Georgia Conference in Topology (to appear).

3. J. W. Milnor, On the cobordism ring $\Omega^{*}$ and a complex analogue. I, Amer. J. Math. 82 (1960), 505-521. MR 22 \#9975.

4. R. E. Stong, Notes on cobordism theory, Princeton Univ. Press, Princeton, N. J., 1969 ,

5. H. Toda, p-primary components of homotopy groups. IV: Compositions and toric constructions, Mem. Coll. Sci. Univ. Kyoto Ser. A Math. 32 (1959), 297-332. MR 22 \#1906.

The University of Virginia, Charlottesville, Virginia 22903 